\title{
La construction du risque au prisme territorial : dans l'ombre de l'archétype alpin, les avalanches oubliées de moyenne montagne
}

\author{
Florie Giacona ${ }^{1, *}$, Nicolas Eckert ${ }^{2}$ et Brice Martin ${ }^{3}$ \\ ${ }^{1}$ Géohistoire, Université de Haute-Alsace, Centre de recherche sur les économies, les sociétés, les arts et les techniques \\ (Cresat), Mulhouse, France \\ ${ }^{2}$ Géophysique, Irstea / Université Grenoble Alpes, UR ETNA, Saint Martin d’Hères, France \\ ${ }^{3}$ Géographie, Université de Haute-Alsace, Centre de recherche sur les économies, les sociétés, les arts et les techniques \\ (Cresat), Mulhouse, France
}

Reçu le 29 décembre 2015. Accepté le 6 avril 2017

L'appréhension des risques fondée sur le couple aléas/vulnérabilité est constamment enrichie d'approches montrant que la perception de ce couple canonique varie selon les acteurs engagés, les agendas politiques, etc. Dans cet article, les auteurs mettent l'accent sur la variabilité territoriale, liée notamment à la construction sociale d'un imaginaire de ces territoires. Utilisant le cas emblématique des avalanches, associées dans les imaginaires aux hautes montagnes, les auteurs montrent comment les imaginaires territoriaux ont contribué à rendre invisible le phénomène dans les moyennes montagnes (ici, dans les Vosges) - et ce en dépit d'accidents récurrents, ce qui conduit à une prise en charge institutionnelle insuffisante. Participant du processus de dévoilement du problème, cet article pointe une prise en charge du risque par les usagers des montagnes - mais qui n'est pas (encore) de nature à changer fondamentalement l'appréhension et la gestion du phénomène.

La Rédaction

Résumé - En France, le risque d'avalanche reste associé à la haute montagne, mais semble oublié dans les espaces de moyenne montagne. À partir des exemples de l'archétype alpin et du massif vosgien, cet article discute les mécanismes sociohistoriques ayant abouti à cette situation. Le phénomène a été appréhendé par et pour la haute montagne, qui bénéficie de ce fait d'un système de prise en compte complet du risque. À l'inverse, la faible visibilité du phénomène en moyenne montagne conduit à une approche édulcorée. Dans un contexte d'absence de construction historique de la vulnérabilité dans le massif vosgien, l'occurrence d'accidents liés aux pratiques récréatives hivernales est toutefois l'occasion d'une (re)découverte de l'avalanche. Ainsi, l'article met clairement en évidence l'importance du prisme territorial dans la mécanique de construction du risque.

Mots-clés : avalanche / risques / construction du risque / territoire / massif vosgien

\begin{abstract}
Risk construction and territory: in the shadow of the Alpine archetype, the forgotten avalanches of medium-high mountains. All mountain ranges in France are subject to avalanche risks. Yet these remain associated with high mountains and are overlooked for lower mountain ranges. Based on the case of the alpine archetype and of the Vosges Mountains, this article discusses the social and historical mechanisms that generated this situation. Literature, as well as the process of scientific and institutional knowledge construction are responsible for the high mountain - avalanche paradigm that still prevails today. Addressed for high mountains, the avalanche phenomenon is visible only in these areas. Tragic events and the emergence of tourism issues led to the development of a comprehensive risk management system, with risk being defined through its hazard components (extension, magnitude, etc.). In medium high mountains on the contrary the phenomenon was overlooked and consequently acquisition of specific knowledge was neglected. The framework developed for alpine areas could therefore not be transposed, which led to a softer, "low cost", risk management system. In these areas suffering from a lack of vulnerability identification and construction, the emergence of winter recreational practices has fostered the
\end{abstract}

\footnotetext{
*Auteur correspondant : florie.giacona@uha.fr
} 
need to (re)discover and take into account avalanche risks. All in all, this article clearly highlights the importance of the territorial prism in the process of risk construction.

Keywords: avalanche / risk / risk construction / territory / Vosges mountains

\section{Introduction}

\section{L’avalanche, un risque territorialisé}

Selon la doctrine nationale française d'établissement des plans de prévention des risques (PPR) naturels (Garry et al., 1997), «l'avalanche est un risque inhérent à la montagne » (MEDDE, 2015). De fait, quoique variables d'un massif à l'autre, phénomène et risque concernent l'ensemble des massifs montagneux du territoire métropolitain. Pourtant, la problématique reste manifestement pensée à travers la haute montagne. Ainsi, les médias nationaux ne relaient qu'exceptionnellement des événements survenus en moyenne montagne et le risque ne fait que peu l'objet d'une prise en compte institutionnelle dans ces territoires. À cet égard, l'exemple du massif vosgien est éclairant. Les phénomènes avalancheux qui s'y produisent sont certes d'intensité et de conséquences socioéconomiques moindres qu'en haute montagne. Les sites d'avalanches vosgiens présentent des caractéristiques qui les distinguent de ceux de haute montagne. D'altitude et d'ampleur plus modestes, ils se particularisent également par la présence d'un couvert forestier important remontant jusque dans les zones de départ. Conséquemment, les phénomènes ne prennent que peu voire pas du tout la forme d'écoulements en aérosol. Toutefois, l'activité avalancheuse est significative dans le massif vosgien, et le risque non négligeable en termes de dommages corporels : une dizaine de décès lui sont imputables depuis les années 1970, ce qui en fait l'un des risques naturels les plus meurtriers en Alsace (Giacona et al., 2017). L'encadré permet d'appréhender plus avant la réalité matérielle du risque d'avalanche dans le massif, en particulier ses caractéristiques physiques et sociales principales.

\section{Le risque, une construction sociale}

Cet article considère le risque comme une construction sociale à l'interface d'interrelations complexes entre les dimensions sociale, spatiale voire territoriale, et temporelle ${ }^{1}$. Dans cette perspective, le risque n'est pas

\footnotetext{
${ }^{1}$ Les idées développées dans cet article ont émergé au cours de la thèse de F. Giacona "Géohistoire du risque d'avalanche dans le massif vosgien. Réalité spatio-temporelle, cultures et représentations d'un risque méconnu» soutenue en 2014 à l'Université de Haute-Alsace. Celle-ci visait à comprendre le paradoxe entre la réalité du risque d'avalanche dans le massif vosgien et son occultation. Dans cet article, on étend de façon originale l'analyse au cadre plus large de la comparaison haute / moyenne montagne.
}

l'avalanche elle-même mais « sa forme sociale projetée » (Martinais et al., 2006). Il prend forme à travers le processus d'identification, de nomination et de définition, et de mise en œuvre d'actions de prévention et protection. Il ne peut donc y avoir risque sans existence d'une conscience du risque, « de la population exposée, [...] d'un groupe extérieur ou [...] de l'ingénieur ou du chercheur »(Peltier, 2005), laquelle s'inscrit dans un contexte socio-spatial donné. Plus qu'un « simple support au risque »(Rebotier, 2010), l'espace constitue une « composante intrinsèque » du risque (Veyret, 2003): le risque façonne l'espace tandis que l'espace participe de la construction des risques. De ce fait, le risque ne peut s'envisager indépendamment des rapports à l'espace et des modes d'occupation du territoire. Son émergence est intimement liée aux connaissances et représentations mobilisées par les acteurs sociaux, ainsi qu'aux relations dialectiques entre représentations, pratiques sociales, phénomène physique et espace.

\section{Mettre à jour les mécanismes de la (non) construction du risque}

L'ancrage territorial des avalanches en haute montagne s'explique notamment par des représentations socialement partagées du phénomène avalanche et de la montagne. Les Alpes constituent le symbole par excellence de la montagne, et l'avalanche apparaît comme une caractéristique incontournable de la haute montagne. Au contraire, l'alliance entre patrimoine naturel et patrimoine culturel définissant la moyenne montagne l'éloigne fortement de l'archétype alpin et cadre mal avec l'idée de massif avalancheux. Le risque d'avalanche concourt ainsi à la construction du territoire en contribuant à dessiner et à définir la « vraie » montagne. Dans cet article, la moyenne montagne est définie au sens de volume montagneux indépendant et non d'étage altitudinal. Il est donc question des massifs de moyenne montagne. Toutefois, pour des raisons de commodité de langage, nous utiliserons le terme «moyenne montagne». La moyenne montagne correspond ainsi, dans l'espace français, au Jura, au massif central et au massif vosgien.

L'objectif de cet article est d'étendre l'analyse à la mise en lumière des mécanismes sociaux et historiques ayant abouti, malgré sa réalité matérielle, à la non construction du risque avalanche dans les territoires de moyenne montagne, en prenant le massif vosgien en tant qu'exemple illustratif. Pour ce faire, on replacera les représentations sociales dans leurs temporalités et 
Encadré. Réalité matérielle du risque avalanche dans le massif vosgien

Le massif vosgien, situé dans le nord-est de la France, s'étend sur 150 kilomètres pour une largeur comprise entre 20 et 60 kilomètres et des altitudes inférieures à 1500 mètres. Bien qu'il s'agisse d'un massif d'extension et d'altitude modestes, les avalanches y sont un phénomène courant et des événements de grande ampleur n’y sont pas à exclure. Les avalanches se produisent préférentiellement dans les cirques glaciaires situés dans la partie méridionale du massif (les Hautes Vosges). Cette localisation s'explique par la combinaison de facteurs nivo-météorologiques et géomorphologiques. Du fait de son orientation (nord-nord-est - sud-sud-ouest), le massif est perpendiculaire aux vents dominants d'ouest qui balayent les chaumes sommitales. Les versants est des cirques glaciaires, surmontés d'étendues planes et déboisées, bénéficient d'une suralimentation neigeuse, qui constitue un élément favorable à la formation de corniches et d'avalanches dans ces sites.

Une étude géohistorique détaillée combinant capitalisation d'informations historiques et études spatiales des sites d'avalanche a permis de recenser plus de 700 événements survenus depuis la fin du XVIII ${ }^{\mathrm{e}}$ siècle (Giacona et al., 2017). La chronologie brute montre que les événements recensés restent sporadiques jusqu'au milieu du $\mathrm{XX}^{\mathrm{e}}$ siècle puis augmentent très nettement à partir des années 1990. En effet, jusqu'au milieu du XX $\mathrm{X}^{\mathrm{e}}$ siècle, les événements dont on a gardé la trace correspondent presque exclusivement à des avalanches de forte intensité et/ou ayant causé des dommages. Ensuite, à l'inverse, l'intérêt se porte progressivement vers tous les phénomènes avalancheux qui se produisent. La nature des dommages occasionnés par les avalanches a également évolué au cours des deux derniers siècles. Au XIX ${ }^{\mathrm{e}}$ siècle et durant les premières décennies du $\mathrm{XX}^{\mathrm{e}}$ siècle, une quinzaine d'événements avec impact sur bâtiments ont été recensés. Certains d'entre eux ont entraîné un nombre important de victimes (jusqu'à dix décès). Néanmoins, près des deux tiers des événements enregistrés ayant causé des dommages corporels (71 au total) ont eu lieu depuis le début des années 1990. Cet accroissement des dommages humains s'explique en partie par l'évolution de la fréquentation du massif vosgien, et donc de la nature des victimes. En effet, aux XVIII ${ }^{\mathrm{e}}$ et $\mathrm{XIX}^{\mathrm{e}}$ siècles, les dommages humains ont concerné essentiellement des personnes qui se trouvaient dans les habitations impactées, tandis que depuis le $\mathrm{XX}^{\mathrm{e}}$ siècle il s'agit exclusivement de victimes pratiquant des activités récréatives hivernales. En parallèle, les dommages fonctionnels (coupures de voie de circulation, une cinquantaine au total), étroitement liés à la viabilité hivernale, apparaissent au milieu du $\mathrm{XX}^{\mathrm{e}}$ siècle, période à partir de laquelle les routes de montagne sont empruntées en hiver. Enfin, les caractéristiques physiques et bioclimatiques du massif rendent les dommages environnementaux significatifs, en particulier pour les peuplements forestiers: près de 80 événements dommageables ont été recensés, parmi lesquels une douzaine a causé des dégâts majeurs (plusieurs hectares de forêt détruits).

Ces chiffres peuvent paraitre relativement faibles par rapport aux dommages causés par les avalanches au niveau national, en particulier dans les massifs de haute montagne des Alpes et des Pyrénées. Ainsi, alors que depuis l'hiver 1971-1972, on compte au total une dizaine de décès par avalanche dans le massif vosgien liés à la pratique d'activités récréatives, la moyenne nationale tourne autour d'une trentaine de décès par an. Néanmoins, à l'échelle régionale ils sont loin d'être négligeables. En outre, de telles comparaisons «brutes» sont à manier avec prudence car la fréquentation (notamment sur le plan quantitatif) et les étendues concernées par le risque d'avalanche sont sans commune mesure entre le massif vosgien et la haute montagne, notamment alpine. Aussi, si les caractéristiques physiques de l'activité avalancheuse (altitude, nature du sol dans les couloirs d'avalanche, nivo-climatologie, taille, intensité et fréquence des événements, etc.) et de la vulnérabilité sont indubitablement différentes dans les deux types de milieux, il n'est pas certain que le risque soit significativement plus faible, au niveau individuel (donc du pratiquant), dans le massif vosgien que dans les massifs de haute montagne.

contextes socioculturels particuliers. Plus largement, nous identifierons les résistances, freins et blocages à l'identification et à la reconnaissance sociale du risque dans ces espaces - deux éléments clés du processus de construction. Nous pensons en effet que les relations entretenues par les sociétés avec le phénomène avalanche et l'espace dans lequel il advient, ainsi que les héritages issus de ces relations, participent de la compréhension de la situation actuelle.

Nous utilisons trois approches complémentaires en faisant dialoguer les échelles spatiales et temporelles d'analyse. La première entend saisir l'origine de l'ancrage territorial de l'avalanche, conduisant à une association plus ou moins consciente du phénomène au milieu alpin, et, en reflet, à son occultation en moyenne montagne. La deuxième, centrée sur la construction scientifique et institutionnelle du risque d'avalanche, vise à éclairer les mécanismes complexes ayant conduit à l'existence d'un système de gestion à deux vitesses, «complet et abouti » en haute montagne, partiel et édulcoré en moyenne montagne. La dernière section dresse le tableau d'une non-émergence de scènes locales du risque dans le massif vosgien, résultant notamment d'une absence de construction collective de la vulnérabilité. Cette dernière évolue toutefois depuis peu vers une prise en compte balbutiante du risque liée aux pratiques récréatives hivernales. 


\section{L'avalanche, un objet « inventé » à travers les Alpes}

Obéissant aux lois de la physique, le phénomène de glissement d'une masse de neige existe de manière «indépendante de notre propre volonté» (Berger et Luckmann, 2006). Il faut donc à la fois le lier et le distinguer de l'objet avalanche, qui est construit socialement et historiquement par et pour la haute montagne. Cette association étroite de l'avalanche à un espace bien déterminé émerge d'abord dans la littérature, puis est renforcée par les services institutionnels en charge de la gestion du risque et par les scientifiques, de sorte qu'elle perdure encore aujourd'hui.

\section{L'émergence de l'association avalanche-haute montagne via la littérature}

La littérature réunit les objets «avalanche » et « haute montagne », de sorte que leur association devient emblématique et participe de l'imaginaire et des représentations. En effet, dès l'Antiquité la figure de l'avalanche émaille les récits et s'inscrit dans un espace particulier, celui de la haute montagne, principalement alpine. Le phénomène est d'abord associé aux passages permettant de traverser les Alpes et aux dangers caractéristiques des montagnes - notamment helvétiques. L'avalanche devient également figure iconographique, souvent sous la forme d'une «pelote», reprise par la littérature savante. $\mathrm{Au}$ XVIII ${ }^{\mathrm{e}}$ siècle, elle est «une attraction attendue», élément significatif du paysage montagnard lors des courses estivales (Rabusseau, 2007), avant de participer de la dramaturgie accompagnant les récits de conquête de la haute montagne enneigée, dans les ouvrages de R. FrisonRoche ou encore de R. Messner.

$\mathrm{Au}$ contraire, ce n'est qu'exceptionnellement que la littérature met en récit des phénomènes survenus en moyenne montagne, et il ne s'agit alors «que» de littérature régionale. Quelques romans de la fin du XIX et du début du $\mathrm{XX}^{\mathrm{e}}$ siècle évoquent des avalanches vosgiennes, décrivant de manière romancée des faits vraisemblables, à défaut de phénomènes qui se soient réellement produits. L'abbé Sifferlen (1909) décrit par exemple une avalanche survenue en février 1895, et qui correspond à un événement connu et documenté. Par ailleurs, quelques guides édités à la même époque citent ponctuellement l'occurrence d'avalanches survenues dans le massif vosgien ${ }^{2}$. Plus récemment, le roman régionaliste de G. Gauchet, L'avalanche (2013), met en scène un accident par avalanche. Il n'est toutefois pas

\footnotetext{
${ }^{2}$ Il s'agit du guide proposé par Jean Bresch (1871) et du «guide Mundel» (Fritsch, 1932). Charles Grad (1889) mentionne également une avalanche dans le secteur du Hohneck dans L'Alsace, le pays et ses habitants.
}

certain que cet ouvrage participe de la culture du risque dans le massif vosgien, car la correspondance avec des faits réels n'est pas explicite et que, par ailleurs, cela supposerait que le lecteur envisage cette réalité.

D'une fonction essentiellement ornementale, l'avalanche alpine acquiert une réalité autonome en devenant objet d'études savantes à partir de la Renaissance. Entre les $\mathrm{XVI}^{\mathrm{e}}$ et $\mathrm{XVIII}^{\mathrm{e}}$ siècles, les naturalistes s'attachent à comprendre et catégoriser le phénomène. Les avalanches sont dès lors résolument inscrites dans le paysage alpin, de sorte que cette association "domine l'imaginaire au détriment des autres massifs d'Europe» (Rabusseau, 2007). De fait, si après avoir été craint, le massif vosgien est découvert et exploré par les savants - botanistes, géologues et minéralogistes - à partir du $\mathrm{XVI}^{\mathrm{e}}$ siècle, ces derniers ne sont, a priori, pas intéressés par le phénomène avalanche. Exception à ce tableau, E. Collomb (1847) s'est attaché au milieu du $\mathrm{XIX}^{\mathrm{e}}$ siècle à décrire et classifier les phénomènes avalancheux vosgiens ${ }^{3}$. Ce sont toutefois les reliques de la dernière glaciation qui constituaient son principal objet d'étude.

Par ailleurs, l'expérience directe de l'avalanche n'a guère pu contribuer à l'attrait du paysage vosgien. En effet, jusqu'au tournant $\mathrm{du} \mathrm{XX}^{\mathrm{e}}$ siècle, explorations scientifiques et pratiques touristiques restent majoritairement estivales. Seuls des dépôts avalancheux ont de ce fait pu être observés à la fin du printemps ou au début de l'été. Les observations effectuées par E. Collomb sont ainsi datées du mois d'avril. Sur ce point, la situation est identique en haute montagne, pour laquelle R. Rabusseau (2007) relève que les naturalistes du XVIII $^{\mathrm{e}}$ siècle évitaient «soigneusement la saison des neiges».

\section{Les institutionnels suivent: l'association avalanche-haute montagne se renforce}

L'avalanche a d'abord été un objet d'étude savant. Elle a ensuite été progressivement prise en compte par les institutionnels et les scientifiques. Suite à l'occurrence de nombreux événements sur l'ensemble du territoire français dans la seconde moitié du XVIII ${ }^{\mathrm{e}}$ siècle et les premières décennies du $\mathrm{XX}^{\mathrm{e}}$ siècle, un lien est clairement établi entre déboisement et catastrophes «naturelles », en particulier les inondations (Veyret, 1943 ; Martin, 1996). Les autorités nationales se saisissent donc du reboisement des montagnes, jugé désormais nécessaire à un moment où elles ont les moyens d'appliquer une telle politique. De cette préoccupation résulte notamment le Code forestier promulgué en 1827 . Notons que le rôle de la forêt de montagne avait été questionné dès le XVIII

\footnotetext{
${ }^{3}$ En 1847, E. Collomb publie dans le Bulletin de la société géologique de France une lettre sur les neiges dans les Vosges à l'attention de $\mathrm{M}$. le secrétaire pour l'étranger.
} 
siècle (Peltier, 2005), mais jusque-là, épisodes révolutionnaires et impériaux faisaient passer la question au second plan (Garnier, 2002).

Les reboisements se poursuivent sous le Second Empire et la Troisième République, avec le vote des lois du 28 juillet 1860 sur le reboisement des montagnes et du 4 avril 1882 sur la restauration et la conservation des terrains de montagne. Les services de la restauration des terrains en montagne (RTM) engagent des travaux de reboisement, de génie civil et de génie biologique dans des zones exposées aux avalanches, aux risques torrentiels et aux mouvements de terrain (Combes, 1989; Besson, 2005). Ils n'interviennent, toutefois, que dans les Alpes et les Pyrénées (Richard et al., 2015) et, encore aujourd'hui, aucun d'entre eux n'est implanté en moyenne montagne. À cet égard, A. Peltier (2005) souligne le fait que le paradigme du déboisement est né dans les Alpes avant d'avoir été "importé» dans les Pyrénées. Le massif pyrénéen étant considéré comme un territoire de montagne, «les forestiers ne [voyaient] pas pourquoi la torrentialité n'y serait pas, [à l'instar des Alpes,] une conséquence du déboisement». Sauf exception, cette transposition ne concernant pas les massifs de moyenne montagne, une distinction institutionnelle entre les deux types de milieux montagnards est donc établie dès cette époque. Est-elle liée au fait que, du point de vue des forestiers, les massifs de moyenne montagne ne constituent pas de "véritables » territoires de montagne? Ou bien résulte-t-elle d'une absence de connaissance des phénomènes qui s'y produisent? Les conséquences présumées du déboisement n'y sont pourtant pas passées sous silence. Ainsi, la commune haut-rhinoise d'Orbey demande en mars 1863 à être soumise au reboisement obligatoire en espérant qu'il aura un effet sur la régularisation du régime des eaux de la Weiss ${ }^{4}$. Plus généralement, suite à la circulaire interministérielle du 25 avril 1821 demandant aux préfets d'étudier l'influence des défrichements sur les climats dans leur département et d'en rendre compte, les inspecteurs des forêts en poste en Alsace établissent un lien direct entre déboisements et problèmes d'érosion et d'inondations ${ }^{5}$. Et déjà, à la suite d'une multiplication des catastrophes naturelles dans la seconde moitié du XVIII $^{\mathrm{e}}$ siècle, le préfet du Haut-Rhin avait en 1801 préconisé un programme de reboisement ${ }^{6}$.

Dans ce contexte, le savoir institutionnel sur les avalanches se construit par l'étude de phénomènes alpins. Ainsi, l'ingénieur RTM P. Mougin met en place en 1899 un système d'enregistrements systématisés des

\footnotetext{
${ }^{4}$ Ministère des Finances, administration des forêts, mars 1863 : Reboisement des montagnes (loi du 28 juillet 1860), compte rendu des travaux de 1862, Paris, Imprimerie impériale.

${ }^{5}$ Archives départementales du Haut-Rhin (ADHR), 7P 44.

${ }^{6}$ Archives départementales du Haut-Rhin (ADHR), 7P 48.
}

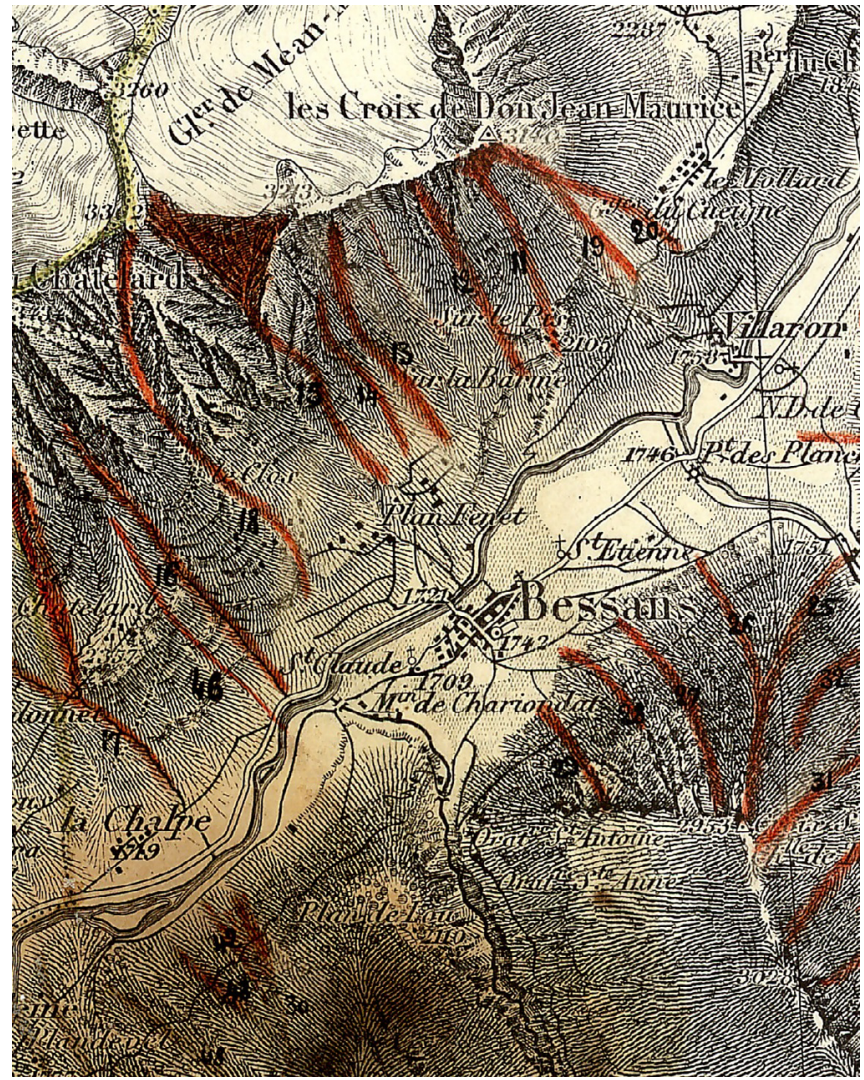

Fig. 1. La cartographie du risque via le phénomène en haute montagne.

Tracé des couloirs d'avalanche de l'EPA en 1929, toujours suivis en 2015. Commune de Bessans, Savoie.

Source: Fond de carte du service géographique de l'armée, levée 1864, révision 1895.

avalanches, l'actuelle enquête permanente sur les avalanches (EPA), qui a d'abord concerné la Savoie (Mougin, 1922) avant d'être étendue progressivement à l'ensemble des Alpes puis aux Pyrénées (Ancey, 2006; Fig. 1). Parallèlement, reflétant l'organisation nationale des services RTM, les avalanches de moyenne montagne passent inaperçues, en particulier celles ayant endommagé des habitations situées en fond de vallée au tournant du XIX ${ }^{\mathrm{e}}$ siècle dans le massif vosgien (Encadré).

\section{Les géosciences parachèvent la construction... et oublient le territoire}

En se saisissant de la question, les géographes instituent les avalanches comme objet d'étude scientifique dès le début du $\mathrm{XX}^{\mathrm{e}}$ siècle, époque marquée par une prégnance de la géographie physique. L'étude du risque d'avalanche s'effectue donc via le phénomène, ses causes et ses effets ${ }^{7}$. Ainsi A. Allix (1925) segmente la

\footnotetext{
${ }^{7}$ D'une manière générale, la géographie française a appréhendé le risque à partir de l'aléa jusqu'aux années 1970 (Veyret et Reghezza, 2005).
} 
« vie » d'une avalanche entre zone de départ, zone de course et zone d'arrivée. Sans surprise, ces premières études portent sur un espace bien défini, la haute montagne, et plus particulièrement les Alpes, dans un moment qui voit la construction de l'objet montagne par les géographes grenoblois, pour lesquels le massif alpin est le modèle par excellence de la montagne (Sgard, 2004). Ce prisme fonctionne toujours à l'heure actuelle. Il explique la difficulté réelle à penser les avalanches comme un objet pertinent en moyenne montagne.

La géographie française cède le pas à des disciplines de plus en plus dures à partir des années 1970 suite à la remise en cause du reboisement comme unique moyen de prévention (Ancey, 2006). L'étude du phénomène avalanche s'effectue alors via la météorologie, la mécanique des fluides, puis l'informatique à mesure que les méthodes de calcul pour l'étude du déclenchement, de la propagation de la neige le long d'une pente, et le dimensionnement des ouvrages de protection se complexifient. Les observations rattachées à des contextes précis cèdent alors le pas à des lois physiques supposées universelles, de sorte que leur lien au territoire de haute montagne est progressivement oublié (Fig. 2).

Sous l'impulsion notamment des scientifiques suis$\operatorname{ses}^{8}$, les sites d'étude de référence en matière d'avalanche ont été implantés au-dessus de $2000 \mathrm{~m}$ d'altitude. Le corollaire à cette focalisation des recherches et des observations in situ des avalanches en haute montagne a été la construction de cadres d'analyse rattachés à cet espace particulier, auquel se rapportent encore les études actuelles. En conséquence, les corpus de référence (ordres de grandeur et/ou valeurs «typiques» auxquels on se compare systématiquement, études pionnières auxquelles on se doit de se référer ultérieurement, etc.) utilisés aujourd'hui pour caractériser le phénomène correspondent à ceux observés en haute montagne. Plus largement, en n'octroyant une visibilité qu'aux seuls phénomènes et événements survenant en haute montagne, cette territorialisation du savoir, largement occultée, a parachevé le paradigme "avalanche égale haute montagne ». Les événements catastrophiques « officiels» recensés et publiés se rapportent ainsi tous aux massifs de haute montagne (Naaim-Bouvet et al., 2015) tandis que les avalanches ayant causé des dommages similaires à la fin du XVIII ${ }^{\mathrm{e}}$ siècle et au milieu du XIX ${ }^{\mathrm{e}}$ siècle dans le massif vosgien restent absentes de cet inventaire (Giacona et al., 2017; Fig. 3 ; Encadré).

In fine, la relative invisibilité des avalanches de moyenne montagne résulte moins d'un défaut de réalité matérielle du phénomène que d'une construction de

\footnotetext{
${ }^{8}$ L'institut fédéral pour l'étude de la neige et des avalanches a été implanté en 1936 au sommet du Weissfluhjoch $(2662 \mathrm{~m}$ d'altitude).
}

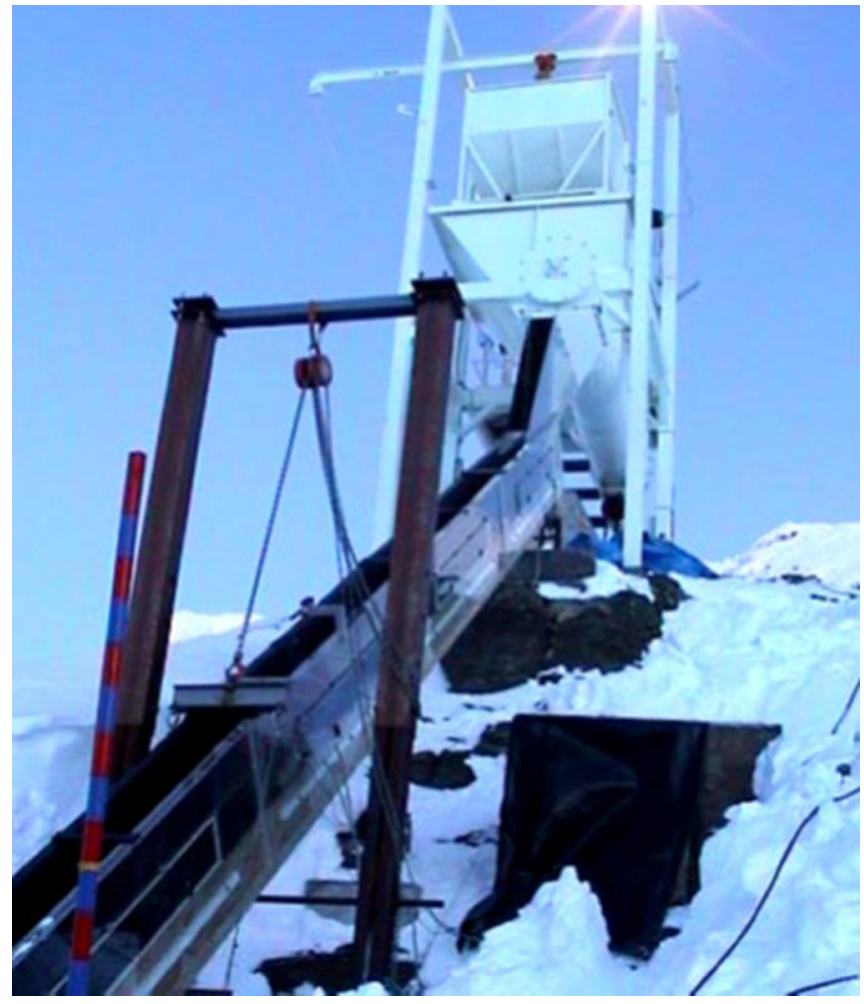

Fig. 2. L'avalanche pensée par et pour la haute montagne. Détermination de lois semi-empiriques à partir d'expériences d'écoulement de neige en canal menées au Col du Lac Blanc, altitude $2830 \mathrm{~m}$, Alpes françaises (Rognon et al., 2008). L'implémentation de ces lois dans des modèles numériques d'écoulement conduit à des outils de quantification du phénomène certes efficaces, mais à la territorialisation implicite. Photo : Irstea

l'objet avalanche intimement lié à la haute montagne. L'évolution des cadres d'étude scientifique n'a pas compensé l'absence de prise en compte institutionnelle de la moyenne montagne. Au contraire, l'acquisition progressive de connaissances territorialisées et l'oubli parallèle de leur territorialisation ont, en débouchant sur des outils de caractérisation du phénomène typés « haute montagne », rendu les avalanches de moyenne montagne d'autant plus invisibles.

\section{Le risque et sa gestion : la genèse d'un système à deux vitesses}

En étant défini par des acteurs fortement focalisés sur le phénomène physique, le risque d'avalanche est confondu avec le phénomène avalanche. Le système de gestion du risque se construit alors principalement autour de la réduction de l'aléa au sein de la haute montagne, lieu de l'avalanche, et, conséquemment, territoire du risque. L'inadéquation de ce schéma à la moyenne montagne conduit à la superposition de 
Extrait de la liste des principales avalanches ayant impacté des bâtiments et survenues en France depuis le $X V^{e}$ siècle. (Naaim-Bouvet et al. 2015)

1419 : 18 morts, 20 habitations détruites à Ristolas (Hautes-Alpes)

1449 : destruction du hameau de Poutuire (Isère), 14 ou 15 morts

1728 : 9 morts dans les hameaux de Bordo et de Coma Esca à Fontpédrouse (PyrénéesOrientales)

1817 : 7 morts suite au passage de 2 avalanches (Les Houches, Haute-Savoie)

1895 : 20 morts à Orlu et Ax-les-thermes (Ariège)

1934 : 9 morts à Bocognano et 37 à Ortiporio (Corse)

1993 : avalanche du Bourgeat (Haute-Savoie), dégâts matériels

1999 : avalanche de Montroc à Chamonix, 12 morts, 14 chalets détruits et 6 endommagés

2008 : dégâts matériels dans le Queyras (Hautes-Alpes) et la vallée de la Tinée (AlpesMaritimes)
Avalanches ayant impacté des bâtiments habités de manière permanente dans le Massif vosgien depuis la fin du XVIII ${ }^{e}$ siècle (Giacona et al., 2016)

1784 : 7 victimes, 5 morts et 1 habitation détruite en Haute-Saône 1789 : 5 morts dans 2 avalanches et 1 habitation partiellement détruite en Alsace

1844 : 10 morts, 1 habitation détruite en Alsace

1942 : destruction d'une habitation en Alsace 1947 : destruction d'une habitation en Lorraine

Fig. 3. Avalanches ayant impacté des bâtiments en France et dans le massif vosgien.

À gauche, un extrait de la liste proposée par Naaim-Bouvet et al. (2015), à droite une liste complémentaire d'avalanches ayant impacté des bâtiments (habités en permanence) de manière similaire (Giacona et al., 2017).

systèmes parallèles, entre des espaces au sein desquels les catastrophes sont «visibles» et des espaces plus «discrets ».

\section{Un objet construit plus tardivement via sa composante aléa}

En s'attachant à la connaissance du phénomène avalanche, indépendamment des dommages que ce dernier occasionne aux enjeux, P. Mougin ouvre la voie à une vision du risque au sein de laquelle prime l'aléa (phénomène naturel associé à une probabilité), confondant parfois les deux. Cette conception prévaut jusqu'à nos jours, notamment en géosciences et chez nombre d'acteurs politico-administratifs (Martin, 2006; Gilbert, 2009) qui négligent ainsi la seconde dimension du risque liée aux enjeux et à leur vulnérabilité. Par ailleurs, de par son appartenance au service RTM et la nature de ses recherches, P. Mougin inaugure le lien qui existe encore aujourd'hui (bien qu'il tende à s'estomper) entre la sphère institutionnelle et les sciences dites «dures». Dans une approche top-down de définition d'une gestion «moderne» du risque, les acteurs institutionnels s'appuient sur les scientifiques et leurs compétences en matière de caractérisation du phénomène physique. Les méthodes de gestion du risque en usage sont ainsi très fortement conditionnées par une «approche ingénieur» dans laquelle le danger est objectivé par la cartographie des zones exposées ainsi que par la réalisation d'ouvrages de protection.

De fait, l'aménagement des zones de montagne se fonde, principalement, sur deux dispositifs de connaissance de l'aléa : l'enquête permanente sur les avalanches, déjà évoquée, et la carte de localisation des phénomènes d'avalanches (CLPA), laquelle s'attache au recensement de toutes les emprises maximales d'avalanche. Ces outils servent de base à l'élaboration des plans de prévention du risque avalanche, définissant le risque en termes de niveau de retour (avalanche centennale en particulier; MEDDE, 2015). Toutefois, en passant directement de la carte des phénomènes à la carte de zonage, sans tenir compte explicitement des enjeux, la cartographie du risque reste "aléa-centrée», et, de ce fait, incomplète (Eckert et al., 2012). Il ne s'agit pas, loin s'en faut, d'une caractéristique française, mais certains pays prennent toutefois en compte à présent la susceptibilité aux 
dommages, de même que des seuils d'acceptabilité sociale du risque dans la cartographie à vocation réglementaire (Arnalds et al., 2004).

De façon similaire, le bulletin d'estimation du risque d'avalanche (BRA), établi quotidiennement par Météo France, intègre très peu le volet «vulnérabilité», se focalisant sur la stabilité du manteau neigeux en fonction des conditions nivo-météorologiques (Giraud, 1993). En outre, le BRA distingue un « risque accidentel », lié à une surcharge ponctuelle extérieure, d'un « risque naturel» faisant référence à un départ spontané. On est donc bien en présence d'un risque qui n'en est pas vraiment un. Notons toutefois que la «contextualisation sociale» intervient, éventuellement, de manière implicite, au niveau du travail du prévisionniste qui transforme les sorties de calcul en un niveau de « risque » (échelle de 1 à 5), de façon à tenir compte de certains comportements des skieurs.

Ces approches aléa-centrées ne sont que très peu contrebalancées par les sciences humaines et sociales puisque peu de travaux s'attachent à la fois au risque d'avalanche et à sa dimension sociale (Busset-Henchoz et Schoeneich, 1996; Granet-Abisset et Brugnot, 2002 ; Boudières, 2008). De fait, de telles études s'inscrivent généralement dans un cadre multirisque embrassant différents types d'aléas et où le risque d'avalanche n'est pas un objet d'étude en tant que tel (Decrop, 1997; Favier, 2005; Barrué-Pastor, 2014). Par ailleurs, en se focalisant, elles aussi, sur la haute montagne, ces études renforcent le paradigme avalanche-haute montagne.

Les deux dimensions du risque restant relativement cloisonnées, et le volet «vulnérabilité » n'étant que peu développé, la construction de l'objet «risque d'avalanche»n'est donc pas pleinement aboutie. Bien que la prise en compte des perceptions et représentations soit de plus en plus intégrée à la prévention, elle reste avant tout destinée aux pratiquants de sports d'hiver (Jarry, 2010), et donc du ressort de l'Association nationale pour l'étude de la neige et des avalanches (ANENA). Cette dernière est, en contrepartie, peu présente sur le volet «risque majeur » concernant, lui, les enjeux humains et matériels situés en fond de vallée (habitations, infrastructures, etc.).

\section{L'importance des catastrophes dans l'impulsion des politiques de gestion}

L'action publique s'est construite a posteriori en réaction à la combinaison de deux facteurs : l'occurrence d'événements dramatiques et des espaces présentant des enjeux économiques forts. Les autorités se sont saisies de la question suite à l'avalanche qui a endommagé, en février 1970, un chalet de l'UCPA à Val d'Isère et causé le décès de 39 personnes. Cet événement a donné lieu à la mise en place d'actions fondatrices: création d'un organisme de liaison et de concertation - l'ANENA -, institution de la CLPA, sensibilisation des propriétaires de chalets, instauration de la surveillance et de l'alerte au risque d'avalanche par Météo France, et mise en place de plans de lutte contre les avalanches par déclenchement préventif - PIDA - en 1972 (Sandraz, 2011).

La catastrophe de Val d'Isère s'inscrit dans un contexte particulier, celui de la conversion de l'économie montagnarde au tourisme, qui s'accompagne d'une modification des enjeux et de l'accroissement de leurs valeurs. Cette mutation socioéconomique entraîne, en corollaire, une modification du rapport entre les territoires et les risques auxquels ils sont exposés. Autrement dit, selon G. Drecop (2004), la «place de choix» occupée par les «dangers naturels du milieu naturel $[. .$.$] dans les représentations sociales de l'espace$ montagnard et dans l'identité de ses habitants » (l'habitude de "vivre avec le risque») cède le pas à une exigence en matière de sécurité. $\mathrm{Si}$, auparavant, l'État français intervenait peu, le risque d'avalanche devient un «risque majeur», qu'il devient «nécessaire» d'objectiver. Un second «moment» constitutif du système actuel de gestion du risque est lié à l'avalanche de Peclerey (Chamonix-Montroc) du 9 février 1999. L'endommagement d'habitations a notamment conduit à la mise en œuvre de l'inventaire des sites sensibles aux avalanches (SSA).

Notons que l'occurrence de dommages économiques est un ressort qui a fonctionné antérieurement. Ainsi, bien que des propositions de techniques paravalanches aient été faites au milieu du $\mathrm{XIX}^{\mathrm{e}}$ siècle afin de protéger l'hôpital militaire de Barèges (Pyrénées), elles n'ont été réalisées qu'à la suite de la catastrophe de 1860 qui a entrainé la destruction dudit hôpital (Ancey, 2006). Autre (contre-)exemple frappant: alors que la catastrophe d'Ortiporio (Corse) de février 1934 a causé entre 37 et 41 décès, elle est passée inaperçue (Deschâtres et al., 2008), sans doute parce que les victimes étaient des résidents permanents, Ortiporio ayant une contribution nulle à l'économie touristique...

Du fait de l'invisibilité du phénomène avalanche en moyenne montagne, l'unique ressort permettant d'élever les massifs de moyenne montagne au rang d'espaces à risque est un drame tel qu'on ne puisse plus occulter voire nier l'existence du phénomène physique et du risque. De fait, la "prise de conscience» institutionnelle d'une problématique "avalanche» dans ces territoires fait suite à un retour d'expérience conjuguant événement tragique et enjeu économique : le décès d'une skieuse sur une piste de ski de la station de Métabief (Doubs) en février 1999, amplement médiatisé, y compris dans les médias nationaux. Météo France se saisit de la question et met en place les «alertes aux coulées de neige» 
(devenues depuis peu «alertes aux coulées de neige et aux avalanches », formule tout de même plus explicite) pour l'ensemble des massifs de moyenne montagne.

\section{Un système de gestion à deux vitesses}

La territorialisation du savoir conjuguée à la présence d'enjeux économiques concourt à donner la primauté aux espaces de haute montagne. L'approche régalienne de type top-down de l'État vers le local conduit à la cohabitation de deux systèmes de gestion du risque d'avalanche. Le premier, pointu et contextualisé, s'appuie, pour la haute montagne, sur tous les instruments de l'action publique (Fig. 4) et sur des acteurs spécifiques, mais confond toujours largement le risque avec l'aléa. Le second, destiné à la moyenne montagne, se caractérise par l'absence de nombreux acteurs, outils et instruments de l'action publique. Les champs de la connaissance du phénomène faisant défaut et les acteurs étant peu spécialisés, la gestion du risque est essentiellement axée sur la mise en place de mesures non structurelles ponctuelles et disparatestelles que l'intervention de pelotons de gendarmerie de montagne en cas d'accident ou la mise en place de plans d'intervention de déclenchement des avalanches (PIDA).

Dans le détail, en moyenne montagne, les gestionnaires doivent composer à la fois avec des outils initialement définis à l'échelle nationale, typés «haute montagne», et avec de moindres ressources, une situation pour le moins inconfortable. Ainsi, les alertes aux coulées de neige et aux avalanches sont diffusées dans un but purement informatif, contrairement aux bulletins d'estimation du risque d'avalanche, conçus comme de véritables outils d'aide à la décision. Ces alertes ne reposent pas sur la stratigraphie du manteau neigeux mais sur l'identification de situations dans lesquelles des avalanches se produisent naturellement: fortes chutes de neige fraîche, et fortes pluies sur un manteau neigeux important. Elles sont alors transmises aux acteurs locaux puis diffusées par le biais des médias. Dans ce contexte, la moyenne montagne ne dispose pas de cartes de vigilance pour avalanches, réservées à la haute montagne. De même, l'extension de l'inventaire des sites sensibles aux avalanches à la moyenne montagne pose également question. Il n'a bénéficié ni de la même attention ni de la même méthodologie qu'en haute montagne (pas de recherches dans les archives dans le cas du massif vosgien par exemple). D'autre part, les critères de classification, définis pour la haute montagne et donc fortement contextualisés, ont été appliqués tels quels à des milieux qui présentent pourtant des spécificités différentes, notamment en termes de morphologie des sites d'avalanches et de modalités de pratique de l'espace (Encadré).

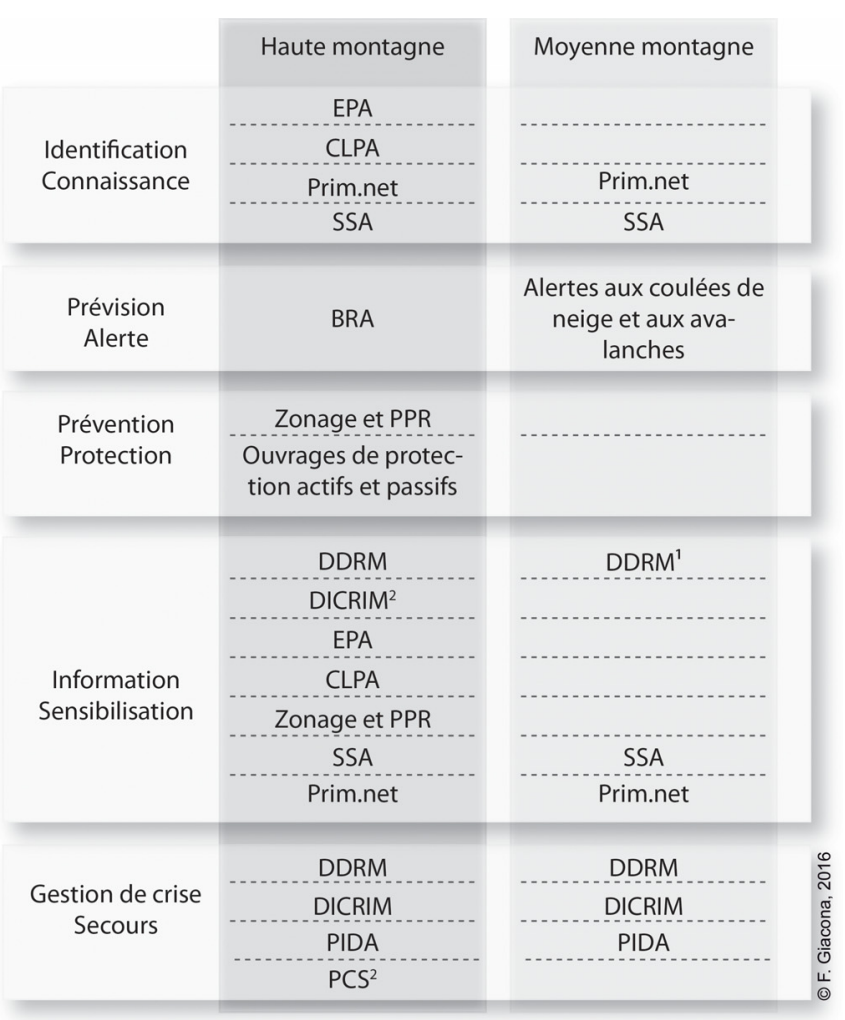

Massif central et Jura uniquement ; sur le territoire du massif vosgien, les DDRM ne contiennent pas d'information concernant les avalanches.

2 Documents non étudiés pour le massif central et le Jura.

Fig. 4. Représentation simplifiée du système de gestion à deux vitesses du risque d'avalanche.

Prim.net: portail institutionnel de prévention des risques majeurs; EPA : enquête permanente sur les avalanches; CLPA : carte de localisation des phénomènes d'avalanche; SSA: sites sensibles aux avalanches; BRA : bulletin d'estimation du risque d'avalanche ; PPR: plans de prévention des risques; DDRM: dossier départemental sur les risques majeurs; DICRIM: document d'information communal sur les risques majeurs; PCS : plan communal de sauvegarde ; PIDA : plan d'intervention de déclenchement des avalanches.

On se trouve ainsi face à une situation paradoxale qui voit, dans un contexte d'absence de reconnaissance et de problématisation du risque d'avalanche en moyenne montagne, la mise en place, malgré tout, d'actions de gestion. Il en résulte nombre d'incohérences ${ }^{9}$. Le dossier départemental des risques majeurs (DDRM) du Puy-deDôme recense ainsi quatre communes soumises au "risque avalancheux», parmi lesquelles ne figure pas

\footnotetext{
${ }^{9}$ La base Gaspar, hébergée par le portail de la prévention des risques majeurs, recense, par commune, les documents d'information préventive ou à portée réglementaire existants. Elle présente des incohérences qui ne sont pas spécifiques au risque d'avalanche. Des études, relatives aux inondations notamment, ont ainsi mis en avant les limites intrinsèques de cette base de données (Douvinet et Vinet, 2012).
} 


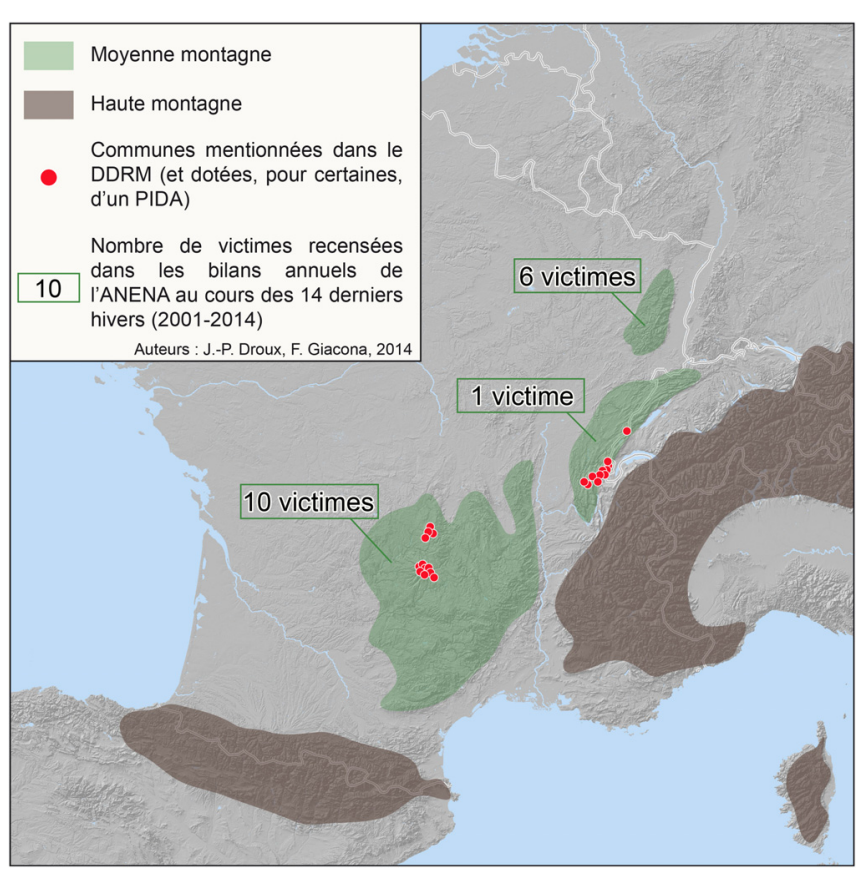

Fig. 5. Prise en compte du risque d'avalanche dans les massifs de moyenne montagne français : identification des communes concernées dans les DDRM et recensement des victimes par l'ANENA.

Mazoires. Pourtant, ce même dossier indique qu'une avalanche y a endommagé une habitation en février 2005, donnant lieu à un arrêté de catastrophe naturelle (CATNAT). De même, alors que Saint-Projet-de-Salers figure sur la carte de l' «aléa avalanche» du DDRM du Cantal, elle n'apparaît pas dans la base Gaspar comme étant soumise à ce risque. Enfin, bien que des alertes aux avalanches et aux coulées de neige soient régulièrement diffusées par la préfecture du Haut-Rhin et bien que soit annexée au plan Orsec départemental une carte de localisation des zones à risque d'avalanche et/ou de chute, le DDRM n'y fait état d'aucun risque d'avalanche, pas plus d'ailleurs que les autres DDRM couvrant le massif vosgien (Fig. 5).

$\mathrm{Ce}$ paradoxe se retrouve jusque dans le guide méthodologique PPR avalanche (MEDDE, 2015). Ce dernier indique que des départements de moyenne montagne sont concernés par le risque, dont le HautRhin et le Bas-Rhin. Toutefois, contrairement au massif central et au Jura, la carte de localisation qui accompagne le texte ne contient aucune commune du massif vosgien. Bien qu'officiellement en charge de l'information préventive sur les risques auxquels les citoyens sont exposés, les pouvoirs publics n'assument donc pas leur rôle dans le contexte du massif vosgien. Ainsi, à la distinction institutionnelle haute-moyenne montagne s'ajoute un second niveau de disparité lié aux conduites différenciées des territoires en matière d'information/ sensibilisation. Un point commun relie toutefois l'ensemble des espaces de moyenne montagne français, l'absence de volet avalanche dans les PPR des communes de ces territoires. Cet état de fait résulte certes pour partie de la faiblesse des enjeux urbanisés soumis aux avalanches en moyenne montagne, mais il démontre également l'oubli de ces espaces dans la construction du risque au niveau de l'État.

\section{Au niveau du territoire vosgien: de l'absence de construction locale de la vulnérabilité aux balbutiements d'une prise en compte du risque}

L'absence de construction du problème avalanche dans le massif vosgien résulte d'une conjonction de l' ' institutionnalisation» de la haute montagne comme territoire du risque avec l'absence locale de construction historique et sociale du risque. Cette dernière s'explique, $\mathrm{du}$ moins en partie, par les interactions entre acteurs locaux, espace et phénomène avalanche, lesquelles ne concourent pas à élever le massif vosgien au rang d'espace à risque. Face au "vide» scientifique et institutionnel, à la faible appropriation et publicisation $\mathrm{du}$ risque, des initiatives locales émergent toutefois, et participent d'une (re)découverte de l'existence de l'avalanche à travers le risque.

\section{Une absence de construction historique et sociale par les populations usagères du massif}

Les pratiquants d'activités récréatives hivernales dans le massif sont actuellement la principale population concernée par le risque. Leurs représentations du massif vosgien reposent sur les dimensions paysagères et du patrimoine naturel, sur l'accessibilité du massif, son caractère ressourçant, sa douceur et sa proximité ${ }^{10}$. Dans ce contexte, le risque d'avalanche ne fait l'objet que d'une faible appropriation. Les pratiquants reconnaissent au mieux l'existence d'un aléa localisé et ponctuel et affichent un manque de bagage historique. Cette absence de «mémoire longue », a fortiori de transmission orale et directe entre plusieurs générations, pourrait s'expliquer en partie par l'importance limitée des enjeux qui étaient exposés par le passé. En effet, bien que des zones

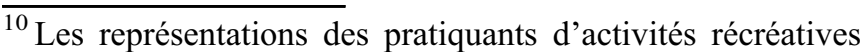
hivernales ont été recueillies par le biais d'un questionnaire auto-administré. Au total, près de 400 questionnaires ont été exploités, complétés en très grande majorité par des personnes vivant dans le bassin environnant du massif. L'ensemble des types d'usages est représenté : ski alpin, ski de fond, raquette, randonnée pédestre, ski de montagne, snowboard, alpinisme, cascade de glace, kite.
} 

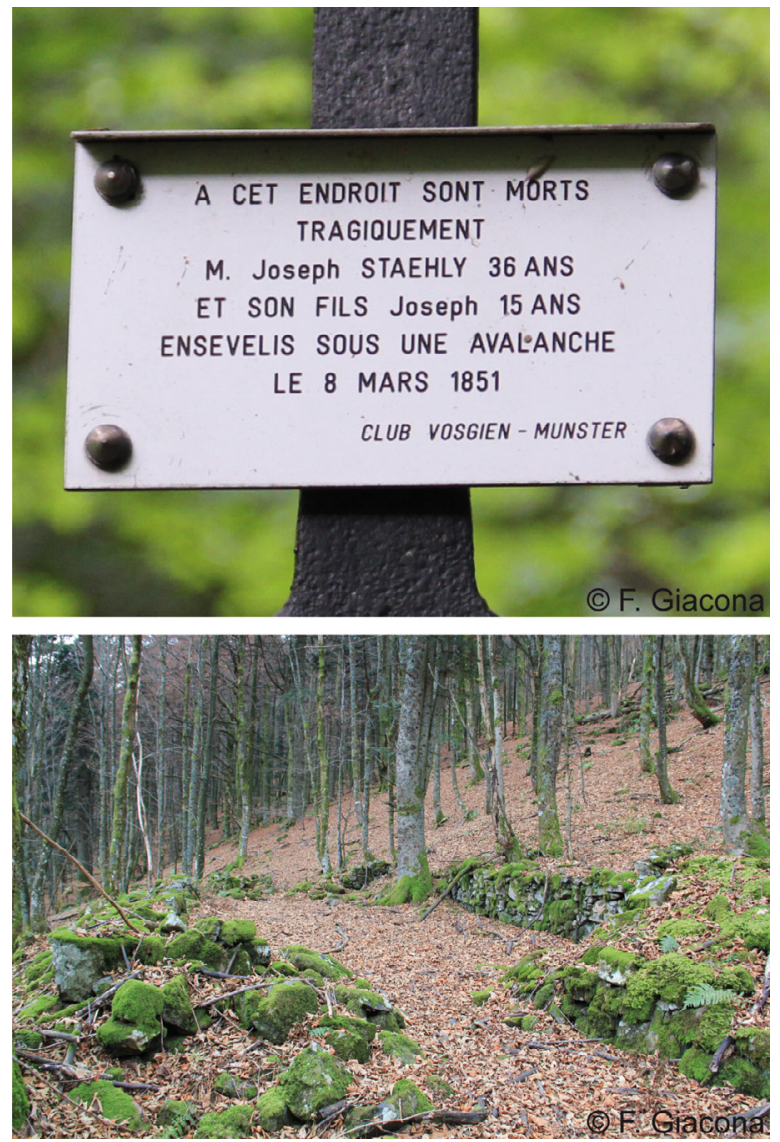

Fig. 6. Exemple d'interaction phénomène avalanche - société au $\mathrm{XIX}^{\mathrm{e}}$ siècle dans le massif vosgien.

En haut: croix indiquant le décès de deux personnes ensevelies sous une avalanche le 8 mars 1851, combe du Leibeilthal, commune de Mittlach.

En bas: ruines d'une ferme d'altitude endommagée par des avalanches durant l'hiver 1826-1827 et en mars 1853, combe du Leibeilthal, commune de Mittlach.

Photos: F. Giacona

potentiellement exposées au phénomène aient fait l'objet de mise en valeur agricole depuis longtemps, les avalanches se produisent, sauf exception, dans des espaces qui, par le passé, étaient peu pratiqués en période hivernale (Fig. 6) ${ }^{11}$. Or, compte tenu de la rudesse des conditions climatiques, les fermes d'altitude n'étaient occupées que durant la saison estivale. De fait, l'augmentation récente du nombre de victimes (Encadré) est vraisemblablement liée à l'essor de certaines pratiques hivernales du massif, désormais parcouru dans sa globalité, y compris dans les secteurs concernés par l'aléa et en-dehors des pistes balisées et sécurisées (Fig. 7). Ainsi, contrairement à certaines communautés

\footnotetext{
${ }^{11}$ Contrairement aux espaces de haute montagne, les avalanches ne menaçaient (et ne menacent) qu'exceptionnellement des zones d'habitation permanente de fond de vallée.
}

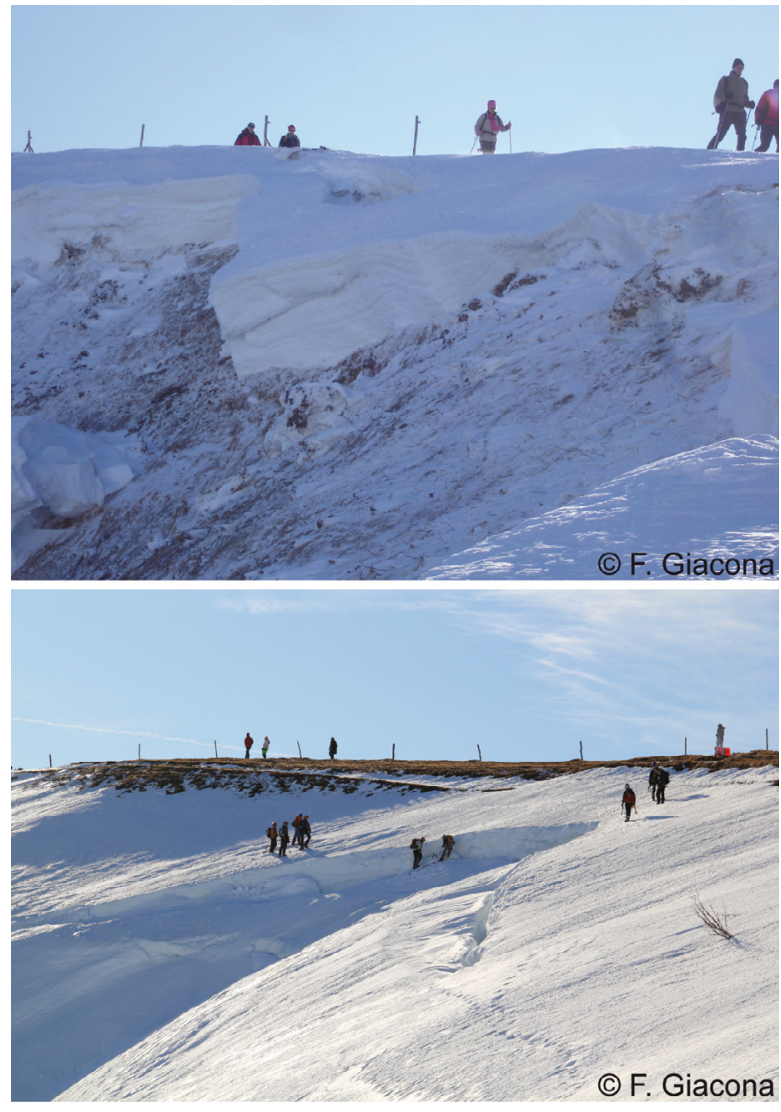

Fig. 7. Nouvelles forme de vulnérabilité dans le massif vosgien: pratiquants d'activités récréatives hivernales en zone de départ d'avalanches dans le secteur du Hohneck.

En haut: randonneurs à raquettes devant une cassure du manteau neigeux en janvier 2009.

En bas : alpinistes devant une cassure du manteau neigeux en décembre 2011.

Photos: F. Giacona

alpines de fond de vallée, le risque d'avalanche dans le massif vosgien n'a jamais fait réellement partie du quotidien. N'ayant pas d'impact structurel sur le système territorial, il n'a sans doute ni influencé fondamentalement les pratiques et les techniques ni permis l'appropriation de la menace par une population exposée. Cela expliquerait l'absence de transmission de savoirs précis territorialisés.

Une analyse approfondie des médias régionaux et nationaux (presse à partir du milieu du XIX $^{\mathrm{e}}$ siècle et journaux télévisés sur la seconde moitié du $\mathrm{XX}^{\mathrm{e}}$ siècle) a montré, d'une part, l'existence de stratégies argumentatives ambiguës, voire de logiques contradictoires évoluant entre la mise en garde contre le risque d'avalanche et sa minimisation, et d'autre part, l'absence d'objectivation du risque (peu de précisions sur la localisation et le phénomène, etc.). Ainsi, le discours médiatique ne compense pas cette absence de construction historique du risque d'avalanche dans le massif vosgien. 


\section{Des acteurs institutionnels locaux historiquement peu sensibles}

Les acteurs institutionnels locaux éprouvent une certaine difficulté à nommer le risque et à en favoriser la compréhension. Une note du préfet du département des Vosges évoque ainsi en avril 1994 la " formation de plaques à vent » ou le fait que la «neige à gobelets [...] rend la surface très instable " mais ne parle pas explicitement d'avalanche ${ }^{12}$. Plus récemment, en janvier 2000, élus, gestionnaires, associations et professionnels de la montagne, réfléchissant de concert à l'élaboration d'un code d'organisation de la pratique de la raquette à neige, relèvent seulement comme principaux problèmes la cohabitation avec les fondeurs et l'impact sur le milieu naturel $^{13}$. On est pourtant dans le contexte d'aprèsaccident de la Crête du Lauzet dans les Hautes-Alpes (ayant concerné des raquettistes en 1998) et, à la fin du même mois de janvier 2000, d'un accident entraînant le décès de deux raquettistes belges dans les Vosges hautrhinoises!

Les gestionnaires locaux souffrent également d'un manque de connaissances, tant du phénomène que du risque, ne cherchant pas réellement à s'appuyer sur des travaux de spécialistes. Ainsi, les quelques démarches entreprises dans ce sens ont procédé d'initiatives individuelles et n'ont obtenu que peu d'appui. Le projet de suivi du manteau neigeux, proposé par un passionné du massif et de sa nivologie, n'a été financé qu'à la suite des accidents de janvier 2000 et reconduit seulement jusqu'en 2004 !

Ce défaut de savoirs explique en partie les ambiguïtés et inconsistances précédemment évoquées en matière de gestion institutionnelle: bien que les alertes soient relayées par la sécurité civile, que deux pelotons de gendarmerie de montagne œuvrent dans le massif et que des exercices de secours soient organisés, le risque n'est pas «dit» et ne fait l'objet d'aucune cartographie systématisée. Il «n'existe» donc pas, et est peu intégré dans l'aménagement du territoire. Tout au plus compte-t-

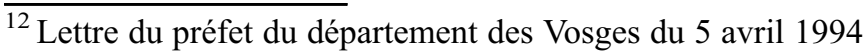
destinée au refuge des Trois Fours, à la ferme-auberge des Trois Fours, à l'hôtel du pied du Hohneck, à la brasserie du Pâquis des fées, au chalet des Amis de la nature (chaume du Haut Chitelet), au chalet universitaire (La Schlucht), au chalet du ski-club de Saint-Maurice-sur-Moselle, au chalet des skieurs de Remiremont, à l'école de ski de la Schlucht, aux mairies de La Bresse, du Valtin et de Stosswihr. Archives communales de Stosswihr: fonds 3, 3H 15.

${ }^{13}$ Cette initiative fait suite à des travaux menés sur la circulation sur les hautes chaumes et leurs versants boisés suivis par les différents acteurs concernés. Archives communales de Soultzeren, fonds 3, $2 \mathrm{H} 9$.
}

on localement quelques panneaux indiquant la présence d'un danger d'avalanche ou de corniches.

\section{Des formes émergentes de savoirs}

Ce tableau plutôt sombre mérite toutefois d'être nuancé. Le risque d'avalanche fait l'objet, à partir des années 2000, d'une nouvelle forme de prise en charge. Face à la faible appropriation et publicisation du risque à l'échelon local, et donc à l'absence d'informations précises, des usagers partagent leurs observations sur des blogs et des sites Internet, de manière plus ou moins structurée. Il s'agit parfois de simples indications concernant l'existence ou non d'activité avalancheuse sur un secteur, plus rarement d'une compilation associant observations, expériences et impressions du terrain, ainsi que résultats de sondages par battage. Messages ou billets interpellent également face, par exemple, à l'absence de bulletin d'estimation du risque d'avalanche dans le massif vosgien. Outre l'intérêt manifeste des données elles-mêmes, ces démarches participent d'une formalisation des connaissances pouvant aboutir à un savoir commun. Néanmoins, bien que parfois partagées au sein de cercles sociaux (groupes de pratiquants de ski de montagne ou d'alpinisme par exemple), ces formes de culture du risque s'inscrivent encore essentiellement dans des logiques individuelles relatives aux expériences d'une vie. Le chemin vers une vraie mémoire collective du risque reste ainsi encore long.

Parallèlement, du côté des acteurs institutionnels et techniques, une légère évolution se dessine vers une considération de la moyenne montagne. En témoigne l'intérêt porté au phénomène avalanche par la préfecture $\mathrm{du}$ Bas-Rhin en vue de l'actualisation prochaine de son dossier départemental des risques majeurs ou de l'identification, certes à moindre frais, de sites sensibles aux avalanches dans le massif vosgien, le massif central et le Jura que l'on a déjà évoqué.

\section{Conclusion}

Résultant de représentations historiquement, socialement et politiquement construites, l'avalanche se pose comme une caractéristique propre à la haute montagne. Tandis que les avalanches de moyenne montagne sont passées inaperçues, les avalanches alpines, «révélées » par la littérature, occupent une place particulière dans le processus de construction du savoir, au point qu'elles dominent encore aujourd'hui les attributs et grandeurs physiques de références utilisés pour décrire le phénomène. Parallèlement, en se construisant dans le contexte particulier de la conversion de la haute montagne en espace de récréation ainsi que par retours d'expérience d'événements dramatiques survenus dans 
les Alpes, les politiques publiques de gestion du risque d'avalanche se trouvent intimement liées aux problématiques rencontrées en haute montagne et aux enjeux socioéconomiques associés. Au contraire, pour la moyenne montagne, la conjugaison d'un marché du tourisme hivernal plus réduit en volume et d'une absence de connaissance du phénomène explique l'établissement progressif d'un système institutionnel de gestion du risque partiel et édulcoré. Enfin, sur la scène locale du massif vosgien, si l'on exclut les balbutiements récents, à tous les niveaux apparaît l'absence d'une conscience et d'une culture du risque. La construction historique et sociale, au sens de la formulation du problème "avalanche», n'a pas eu lieu $\mathrm{du}$ fait d'une absence d'identification collective de la vulnérabilité.

L'analyse s'est focalisée quasi exclusivement sur l'opposition Alpes/massif vosgien mais est aisément généralisable. En effet, l'archétype alpin possède intrinsèquement une ambition universaliste, et presque tout ce qui est vrai pour le massif vosgien s'applique aux autres massifs de moyenne montagne français voire européens, en tout cas à ceux dont les caractéristiques physiques et sociales en sont suffisamment proches. En Suisse, par exemple, si, du fait de l'organisation cantonale, les zones de moyenne montagne ont sans doute été moins «oubliées», la cartographie des zones soumises aux avalanches a bien été réalisée partout sur la base de modèles d'écoulement développés et validés en haute montagne (Salm et al., 1990). En raison des interactions entre le phénomène avalanche et l'espace dans lequel il survient, ainsi que des héritages issus de ces relations, la moyenne montagne ne participe donc pas, aux côtés de la haute montagne, de la «fabrique du risque » (Gilbert, 2003) spécifique à cet aléa. En retour, en ne constituant, au mieux, qu'un fait reconnu ponctuellement et en n'apparaissant pas comme un élément de caractérisation du territoire, le risque d'avalanche ne contribue pas au processus de construction du territoire «moyenne montagne».

Enfin, cette étude, questionnant la mécanique complexe de la construction du risque au prisme territorial, met en évidence des modèles parallèles portés par des enjeux, des savoirs et des représentations différentes, et dont la pertinence dépasse le seul cas des avalanches. Le premier, celui de la haute montagne, est porté par les politiques publiques «de haut en bas» dans une vision centralisée typiquement française. Bien qu'il revête l'idée d'unicité, il exclut de facto certains espaces montagnards. Héritier d'une histoire, il fait la part belle au phénomène dans une vision du risque naturel très «aléa-centrée », d'ailleurs encore largement partagée bien au-delà des frontières françaises. Le second, celui de la moyenne montagne, est porté par la sphère locale à partir des dommages qui rendent l'existence du risque et du phénomène indéniable. À la faveur du développement des techniques de communication, de nouveaux acteurs font leur apparition et se posent «en entrepreneurs de cause» (Neveu, 2015), en s'autosaisissant par exemple de la question d'un « suivi» du manteau neigeux. La réponse institutionnelle, certes encore balbutiante, s'effectue alors davantage « de bas en haut», mettant en lumière l'inadéquation, pour la moyenne montagne, d'outils que l'on a oublié d'adapter à tous les territoires de montagne. A contrario, ce second modèle témoigne du caractère inachevé de la construction de la vulnérabilité en haute montagne. Dans aucun des deux types de milieux, la construction du risque n'est donc réellement achevée, de sorte que le champ reste largement ouvert pour mieux répondre à la demande de sécurité.

\section{Références}

Allix A., 1925. Les avalanches, Revue de Géographie Alpine, $13,2,359-423$.

Ancey C. (Dir.), 2006. Dynamique des avalanches, Lausanne, Presses polytechniques et universitaires romandes.

Arnalds P., Jonasson K., Sigurdson S.T., 2004. Avalanche hazard zoning in Iceland based on individual risk, Annals of Glaciology, 38, 285-290.

Barrué-Pastor M. (Ed.), 2014. Cultures du risque en montagne. Le Pays Toy, Paris, L'Harmattan.

Berger P., Luckmann, T., 2006. La construction sociale de la réalité, Paris, Armand Colin.

Besson L., 2005. Les risques naturels. De la connaissance pratique à la gestion administrative, Voiron, Éditions Techni-Cités.

Boudières V., 2008. De la gestion des risques aux risques de leur gestion dans les territoires touristiques de montagne. Modalités de gestion du risque d'avalanches dans les Alpes françaises et facteurs de vulnérabilité associés. Thèse de doctorat, Grenoble, Université Joseph Fourier, Institut de Géographie Alpine, http://hal.inria.fr/docs/00/37/01/62/ $\mathrm{PDF} /$ manuscrit these $\mathrm{V}$ Boudieres.pdf.

Bresch J., 1871. La vallée de Munster et les Vosges centrales. Guide du touriste, Colmar, Eugène Barth libraire-éditeur.

Busset-Henchoz M.-C., Schoeneich, P., 1996. Stratégies traditionnelles face au danger d'avalanches, Comptes rendus du Symposium Interpraevent, Garmisch-Partenkirchen 4, 255-264, www.interpraevent.at/palm-cms/upload files/Publikationen/Tagungsbeitraege/1996_4_255.pdf.

Combes F., 1989. Restauration des terrains en montagne. Du rêve à la réalité, Revue forestière française, 2, 91-106.

Decrop G., 1997. De l'expertise scientifique au risque négocié. Le cas du risque en montagne, Antony, Cemagref.

Decrop G., 2004. La montagne, le hameau et le prophète de malheur. Histoire d'un risque moderne, Ethnologie française, 34, 1, 49-57. 
Deschâtres M., Guardiola S., Goetz D., Eckert N., Latu V., Richard D., 2008. Avalanche meurtrière à Ortiporio en févier 1934 (Haute-Corse), Neige et Avalanches, 123, 2-7.

Douvinet J., Vinet F., 2012. La carte des arrêtés «CatNat» pour les inondations: limites et améliorations possibles, M@ppemonde, 107, 3, 1-17.

Eckert N., Keylock C. J., Bertrand D., Parent E., Faug T., Favier P., Naaim M., 2012. Quantitative risk and optimal design approaches in the snow avalanche field: Review and extensions, Cold Regions Science and Technology, 79-80, 1-19.

Favier R., 2005. Construire sous la menace en milieu de montagne dans la France d'Ancien Régime, in Istituto Internazionale di Storia Economica F. Datini, L'edilizia prima della rivoluzione industriale secc. XIII-XVIII, Atti della Trentaseiesima Settimana di Studi, 26-30 aprile 2004, Prato, Italie, 429-439.

Fritsch L., 1932. Les Vosges et l'Alsace. Guide du Touriste, Strasbourg - Paris, Librairie Istra.

Garnier E., 2002. La politique forestière de la monarchie et les catastrophes naturelles dans les Vosges aux XVIII ${ }^{\mathrm{e}}$ et $\mathrm{XIX}^{\mathrm{e}}$ siècles, in Favier R. (Ed.), Les pouvoirs publics face aux risques naturels dans l'histoire, Grenoble, Publications de la MSH-Alpes, 219-242.

Garry G., Graszk E., Dupuy JL., 1997. Plans de prévention des risques naturels prévisibles (PPR). Guide général, Paris, La Documentation française.

Gauchet G., 2013. L'avalanche, Barr, Le Verger.

Giacona F., Eckert N., Martin B., 2017. A 240-year history of avalanche risk in the Vosges Mountains based on nonconventional (re)sources, Nat. Hazards Earth Syst. Sci., 17, 887-904, https://doi.org/10.5194/nhess-17-887-2017.

Gilbert C., 2003. La fabrique des risques, Cahiers internationaux de sociologie, 114, 1, 55-72.

Gilbert C., 2009. La vulnérabilité : une notion vulnérable ? À propos des risques naturels, in Beccera S., Peltier A. (Dir.), Risques et environnement: recherches interdisciplinaires sur la vulnérabilité des sociétés Paris, L'Harmattan, 26-40.

Giraud G., 1993. MEPRA: An expert system for avalanche risk forecasting, in Armstrong R. (Ed.), ISSW'92. A merging of theory and practice. International Snow Science Workshop, Breckenridge, CO, 97-104.

Grad C., 1889. L'Alsace, le pays et ses habitants, Paris, Librairie Hachette et Cie.

Granet-Abisset A.-M., Brugnot G., 2002. Avalanche et risques. Regards croisés d'ingénieur et d'historiens, Grenoble, Publications de la MSH-Alpes.

Jarry F., 2010. Avalanche. Risque «majeur» et risque «sportif»: des moyens de prévention différents, Risques infos, 26, 11-12.

Martin B., 1996. Les aléas naturels à Vars (Hautes - Alpes, France), le rôle des facteurs naturels et des facteurs anthropiques dans leur occurrence et leur évolution de 1800 à nos jours. Thèse de doctorat, Strasbourg, Université Louis Pasteur.

Martin B., 2006. Expertise et risques majeurs : le point de vue du géographe, Droit de l'Environnement, 142, 314-323.
Martinais E., Morel-Journel C., Duchêne F., 2006. La construction sociale du risque environnemental : un objet géographique?, in Séchet R., Veschambre V. (Ed.), Penser et faire la géographie sociale: Contribution à une épistémologie de la géographie sociale, Rennes, Presses universitaires de Rennes, 173-186.

MEDDE (ministère de l'Écologie, du Développement durable et de l'Énergie), 2015. Plan de prévention des risques naturels-Avalanches. Guide méthodologique, www.ecolo gique-solidaire.gouv.fr/sites/default/files/150928_guide $\%$ 20PPR $\% 20$ avalanches-ao $\% \mathrm{C} 3 \% \mathrm{BBt} \% 202015$-reduit_0. pdf.

Mougin P., Ministère de l'Agriculture, Direction générale des Eaux et Forêts; Eaux et Génie rural, Service des Grandes Forces Hydrauliques, 1922. Études glaciologiques: les avalanches en Savoie, Paris, Imprimerie Nationale, 4, 175-322.

Naaim-Bouvet F., Deschâtres, M., Jarry, F., 2015. Avalanches de neige en quelques chiffres, in Naaim-Bouvet F., Richard D. (Ed.), Les risques naturels en montagne, Versailles, Éditions Quae, 35-53.

Neveu E., 2015. Sociologie politique des problèmes publics, Paris, Armand Colin.

Peltier A., 2005. La gestion des risques naturels dans les montagnes d'Europe occidentales. Étude comparative du Valais (Suisse), de la Vallée d'Aoste (Italie) et des HautesPyrénées (France). Thèse de doctorat, Toulouse, Université Le Mirail.

Rabusseau R., 2007. Les neiges labiles : une histoire culturelle de l'avalanche au XVIII ${ }^{e}$ siècle, Genève, Presses d'Histoire Suisse.

Rebotier J., 2010. La dimension territoriale des risques urbains à Caracas - Proposer un regard critique de géographie des risques à travers la territorialisation, Congress of the Humanities and Social Sciences at Concordia University Canadian Association for Latin and Caribbean Studies, Montréal, Canada, http://hal.inria. fr/docs/00/48/90/87/PDF/Rebotier_CALACS_Contribu tion.pdf.

Richard D., Marco O., Nepote-Vesino R., 2015. Depuis quand s'occupe-t-on du risque naturel en montagne?, in NaaimBouvet F., Richard D. (Ed.), Les risques naturels en montagne, Versailles, Éditions Quae, 36-40.

Rognon P., Chevoir, F., Bellot H., Ousset F., Naaim M., Coussot P., 2008. Rheology of dense snow flows: Inferences from steady state chute-flow experiments, Journal of Rheology, 52, 3, 729-748.

Salm B., Burkard, A., Gubler, H.U., 1990. Calcul des avalanches : une méthode pour le praticien avec des exemples. Note technique de l'Institut fédéral suisse pour l'étude de la neige et des avalanches, http://www.toraval.ch/ traductions/1992SLF.pdf.

Sandraz R., 2011. Restitution des tables rondes, Neige et Avalanches, 135, 12-14.

Sgard A., 2004. Un moment de la construction du savoir sur la montagne: Jules Blache dans «L'Homme et la Montagne » (1934), in Debarbieux B., Fourny M.-C. (Eds), L'effet 
géographique. Construction sociale, appréhension cognitive et configuration matérielle des objets géographiques, Grenoble, Publications de la MSH-Alpes, 37-54.

Sifferlen G., 1909. La vallée de Saint-Amarin : notes historiques et descriptives, tome III, Strasbourg, F. X. Le Roux \& Cie.
Veyret P., 1943. Un centenaire : l'étude sur les torrents des HautesAlpes de Surell, Revue de géographie alpine, 31, 4, 513-524. Veyret Y. (Dir.), 2003. Les risques, Paris, Sedes.

Veyret Y., Reghezza, M., 2005. Aléas et risques dans l'analyse géographique, Annales des Mines, série «Responsabilité \& Environnement », 40, 61-69.

Citation de l'article : Giacona F., Eckert N., Martin B., 2017. La construction du risque au prisme territorial : dans l'ombre de l'archétype alpin, les avalanches oubliées de moyenne montagne. Nat. Sci. Soc. 25, 2, 148-162. 